\title{
La Gestión de los Recursos Humanos: Dos Percepciones Diferentes de Una Misma Realidad
}

\author{
Yolanda Navarro Abal* \\ José A. Climent Rodríguez
}

Resumen:

El área de los Recursos Humanos es un campo de especialización inter y multidisciplinary, por tanto, su evolución, desarrollo y estructura está modulada por la influencia de cada una de ellas. Este trabajo tiene como objetivo general analizar la percepción y actitud que se muestra de ésta en función de la titulación que se esté cursando. La muestra está compuesta por 84 alumnos pertenecientes a la Licenciatura de Ciencias del Trabajo y Grado en Relaciones Laborales y Recursos Humanos y a la Licenciatura de Psicología. Los resultados muestran diferencias entre ambas titulaciones principalmente en lo referente a la concepción del Departamento de Recursos Humanos como un Departamento en Linea o Staff; en lo relacionado con la función de Gestión del Presupuesto y de Compensación, del Desarrollo, de Relaciones Laborales y de Prevención de Riesgos Laborales y de Gestión del Proceso y de los Resultados, y, en cuanto al perfil necesario para ser un buen gestor de los Recursos Humanos, excepto en lo relacionado con competencias profesionales de carácter transversal tales como el Trabajo en Equipo y las Habilidades de Comunicación, en las cuales se equiparan los resultados obtenidos.

Palabras Claves:

Recursos Humano; Percepción; Gestión; Competencias; Interdisciplinariedad; Multidisciplinariedad.

* Dpto. psicología clínica, experimental y social. Universidad de Huelva.

yolanda.navarro@dpsi.uhu.es 
ABSTRACT:

The area of Humans Resources is an interdisciplinary and multidisciplinary specialized domain and, thus, its evolution, development and structure is shaped by the influence of these different areas. The aim of this paper is to analyze the perception and attitude of different university students about Human Resources, studying the relationship between their attitudes and their degree. The sample is made up of 84 students (of the degrees in "Labor Studies" and "Labor Relations and Human Resources", and "Psychology"). The results show differences between students of different degrees, mainly about their beliefs on the Department of Human Resources as a Staff department (in relation with the function of Management of Budget and of Compensation, of Development, of Labor Relations and Prevention of Workplace Hazards and of Management of the Process and the Results), and related to their idea of good agent of Human Resources, except professional competences as "being a good team player" and "communicative skills" (with similar results).

KEYwORDS:

Human Resources; Perception; Management; Competences; Interdisciplinary; Multidisciplinary.

\section{1.- INTRODUCCIÓN}

El área de gestión de los Recursos Humanos (RRHH) ha ido creciendo en importancia en los últimos años. Desde la perspectiva organizacional, ha cobrado interés la adecuada gestión de los RRHH para el buen funcionamiento de las organizaciones. Inicialmente, fueron las empresas de carácter privado las que añadieron valor a este aspecto organizacional y, en la última década, las instituciones públicas están desarrollando planes estratégicos en donde el Departamento de Recursos humanos cada vez tiene un papel más esencial.

La gestión de personas o gestión del talento humano es una de las funciones empresariales que más ha evolucionado en los últimos tiempos. Se puede decir, que la gestión de los recursos humanos ha evolucionado desde una perspectiva implícita e informal a una más explícita o formal, es decir, más sistematizada. Para algunos autores como Gómez-Mejías et al. (2001) esta creciente preocupación por la gestión de los recursos humanos está motivada por los rápidos cambios profundos en el entorno, tanto a nivel organizativo como a nivel individual. A grandes rasgos, estos autores consideran que estos cambios o exigencias se pueden sintetizar en: - relacionados con el entorno (cambio rápido, crecimiento de internet, globalización y diversidad de la fuerza del trabajo, legislación; evolución del trabajo y del papel de la familia, el crecimiento del sector servicios; - relacionados con la organización (posición competi- 
tiva, flexibilidad empresarial, reestructuración organizativa, problema de reducción del tamaño del personal, utilización de equipos de trabajo autodirigidos, el crecimiento de las pequeñas empresas, la necesidad de crear una cultura organizativa fuerte, el papel de la tecnología y la mayor contratación externa de tareas); - de carácter individual (ajuste persona-organización; dilemas éticos y responsabilidad social; delegación de autoridad, productividad, inseguridad en el trabajo y seguridad social)

En este sentido, Besseyre des Horst (1988) nos señala, que los cambios que se han producido en la función del personal van más allá de un cambio de nombre bajo el cual se agrupan las actividades que se realizan dentro de una empresa. Muy al contrario, implica una cambio conceptual de la Gestión de los RRHH. De esta forma, el concepto se modifica de considerar al personal de una organización como un coste a entenderlo como un recurso para la optimización de la empresa. A su vez, este autor resalta que, como consecuencia de esta transformación, la Dirección de los RRHH abandona las perspectiva caracterizada por los resultados a corto plazo, la focalización sobre los resultados, el análisis cualitativo del desempeño, la rigidez y dependencia en las prácticas, para situarse en un punto de vista definido por los resultados a medio o largo plazo, la focalización sobre los medios y resultados de las acciones, el análisis cualitativo de la evaluación del desempeño, la flexibilidad y la autonomía. Es decir, el fomento del empoderamiento de las personas como autogestoras de sus propias decisiones y la dirección de los RRHH como un asesor, supervisor y guía en el desempeño. Otros autores como Albizu y Landaeta (2001) y en lo referente a las funciones del Departamento de RRHH, opinan que éstas han ido evolucionando paulatinamente desde la administración de personal hacia la gestión y desarrollo en ésta área de conocimiento para llegar finalmente a la gestión estratégica de los recursos humanos. Coincidiendo con este argumento, Bohlander et al. (2003) explica que las funciones de recursos humanos han cambiado orientándose a conseguir retos competitivos como la globalización, incorporación de nuevas tecnologías, administración del cambio, desarrollo del capital humano, respuesta del mercadeo y control de costes.

En paralelo a los diferentes modelos y funciones surge otro de los grandes debates que guarda relación con la concepción del Departamento de RRHH. Estamos hablando de la consideración del mismo como un modelo Staff o en Línea. Es obvio que no es conveniente hacer generalizaciones y es importante tener en cuenta que la estructura dependerá de la tipología organizacional y su cultura. No obstante, cada vez existe más tendencia a considerar la dirección de los RRHH como transversal en la que se involucren a todos los departamentos y personas sobre un equipo humano (Cascio, 1998, Gómez-Mejía et al, 1999). Desde este punto de vista pierde sentido la clasificación del departamento de Dirección de personal como mero Staff de la organización y adquiere una dimensión más amplia concebirlo como una unidad estratégicamente implicada en la representación de los servicios propios de la organización (Martin y Delgado, 2008). 
Existe una amplia variedad de funciones reconocidas en relación al área de los RRHH. Para la elaboración de este trabajo hemos optado por la aportada por Martín y Delgado (2008), en la que hacen una categorización en 9 funciones esenciales que se describen a continuación:

1. Función estratégica incluiría el procedimiento estratégico que englobe las actividades de análisis interno y externo de recursos humanos, el diseño, la planificación elaboración de las estrategias a seguir, la alineación estratégica de procesos, el análisis u gestión de la cultura, la gestión del cambio y la globalización o los procesos de internalización de la empresa. Otros autores como Dolan et al. (1999) contemplan las actividades vinculadas con la alineación estratégica de la gestión de RRHH y las relacionadas con la globalización y gestión internacional; Cascio (1995) también incluyen las tareas relacionadas con la gestión del cambio.

2. Función analítica, según González y de Elena (1998), englobaría las actividades de análisis y valoración de puestos de trabajo, la planificación de recursos humanos, el diseño y utilización de sistemas de información de recursos humanos, el análisis y evaluación de otras variables individuales y grupales; análisis de la estructura de la empresa, el diseño y actualización del organigrama o el inventario de recursos humanos.

3. Función de vinculación y desvinculación, incluye las actividades de la política de selección de la empresa conformada por las tareas de reclutamiento, selección, contratación, acogida y socialización así como las actividades llevadas a cabo para la desvinculación de la persona empleada de la organización.

4. Función de desarrollo (Cascio, 1998; Milkovich y Boudreau, 1994; Ivancevich, 1998; Besseyre des Horts, 1988; González y De Elena, 1998) es la relacionada con las tareas de orientación, formación, desarrollo, evaluación del desempeño y planes de carrera.

5. Función de gestión del presupuesto de Recursos Humanos y de compensación. Esta función adquiere diferentes nombres, así algunos autores las denominan de compensación (Werther y Davis, 1991; Milckovich y Boudreau, 1994; González y De Elena, 1998 y Peiró, 1999), otros de retribución (Gómez Mejías et al. 1999) o de evaluación y retribución (Dolan et al., 1999). En esta función se agrupan, fundamentalmente, las actividades relativas al diseño de sistemas salariales y a la administración de salarios, incentivos y beneficios económicos o sociales.

6. Función de Relaciones Laborales (Gómez-Mejiá et al., 1999; Peiró, 1999), de relaciones con sindicatos y empleados (Milkovich y Boudreau, 1994), o de normas y relaciones laborales (González y De Elena, 1998) constituye otro conjunto de actividades características de la dirección de Recursos Huma- 
nos. Werther y Davis (1991), por ejemplo, establecen dos campos distintos para clasificar las tareas englobadas en esta función. Se trata, por un lado, de las relaciones industriales, y se refiere a las actividades de relaciones con los trabajadores, tales como el fomento de la calidad de la vida laboral, y la comunicación y las relaciones con los sindicatos, de otro lado, integraría el grupo de actividades propias de esa interacción.

7. Función de prevención de riesgos laborales, es el grupo de actividades tales como la asistencia sanitaria o la prevención y seguridad en el trabajo. También recibe otras denominaciones, así Ivancevich (1998), habla de función de protección y evaluación; de derechos de los empleados según Gómez y Mejías (1999) o salud y seguridad, según el modelo de González y De Elena (1998) se integran como salud y seguridad. Estas actividades tienen como objetivo no sólo la prevención sino la mejora de la calidad de vida en el trabajo.

8. Función de la gestión del proceso y de los resultados. Se incluyen en esta clasificación todas las acciones relacionadas con la aplicación de la auditoría de Recursos Humanos, la identificación de aspectos no conformes, la intervención sobre ellos, así como aquellas actividades orientadas más específicamente a mejorar la productividad.

9. Función consultora, que según la concepción actual del Departamento de RRHH, es el eje central que engloba el asesoramiento permanente, y, por tanto, podría considerarse transversal a todas las funciones. Entre sus principales tareas incluye la relacionada con la consultoría de comunicación, desde donde se articulan las políticas de comunicación de la organización y se diseñan los planes y acciones de mejora en el área.

Para finalizar, esta pequeña introducción teórica, es necesario detenerse en el aspecto relacionado con el perfil del Gestor de RRHH. Algunos autores como Albizu y Landaeta (2001), Bohlander et al. (2003) y Alles (2008) consideran que las funciones del Departamento de RRHH están asociadas directamente con el perfil del Gestor de los RRHH, así como la estrategia de negocio y a responder a las necesidades internas y del entorno en el cual está inserta la organización para responder a los desafíos competitivos a los cuales se enfrente. Como se ha referido anteriormente, todos estos cambios en el departamento de RRHH han significado también la necesidad de modificar el perfil existente en las personas que, valga la redundancia, tienen que "gestionar personas". Así, se observa, como inicialmente, se encargaban de gestionar sólo aspectos relativos más al coste personal así como a la legislación y fueron tomando relevancia, en este sentido, los profesionales del derecho. Posteriormente, cuando el modelo comenzó a focalizarse en la persona, fueron los profesionales de 
la psicología los que cobraron protagonismo. Actualmente, es evidente que el área de los RRHH es inter y multidisciplinar, y, por tanto, tiene su propia identidad. La visión reduccionista y fragmentaria, sólo puede llevar a la infravaloración de una especialización tan relevante como ésta. Esta circunstancia implica que las personas encargadas de la formación de base deben tener apertura mental y un conocimiento suficiente desde diferentes perspectivas de este campo de trabajo para evitar posibles sesgos y, consecuentemente, la impartición de la formación de una manera inadecuada. El gestor de personas debe contar con un perfil tanto duro, con una formación necesaria, como blando, con una serie de competencias necesarias para su buen desempeño. De esta forma, son los profesionales de la educación los que deben tener en cuenta estas características y, desde los diferentes planes de estudio, apostar por la inter y multidisciplinariedad si se quiere abogar por una formación de calidad.

De esta forma, se suscita la necesidad de investigar sobre la percepción del alumnado sobre este campo de especialización y la influencia de la titulación que cursa. Así mismo, consecuencia de esta inquietud surgen varias preguntas de interés para la reflexión de la mejora de la calidad docente y de la formación del futuro profesional de lo RRHH, itransmiten los docentes una actitud en las diferentes asignaturas demasiado sesgadas a su titulación de origen?, ¿qué parte de responsabilidad tienen los profesores en esta percepción diferente que manifiestan del gestor de RRHH?, ¿es necesario que los profesores se planteen realmente un cambio hacia un pensamiento más interdisciplinar o es realmente este un hecho que enriquece?.

\section{2.- OBJETIVOS}

- Analizar las diferencias contempladas en el alumnado en relación al modelo de Gestión de los RRHH como Departamento en Line o Staff

- Estudiar las diferentes funciones que se asocian al Área de Gestión de los RRHH teniendo en cuenta la titulación de origen del alumnado.

- Comparar las competencias profesionales asociadas al perfil del profesional de los RRHH en función de la Titulación en donde se encuentre matrículado el alumnado.

\section{3.- MÉTODO}

\section{1.- PARTicipantes}

La muestra está compuesta por 48 alumnos pertenecientes a la Licenciatura de Ciencias del Trabajo y Grado en RLL y RRHH de la Facultad de Ciencias del Trabajo y 36 alumnos de la Licenciatura de Psicología que han cursado la asignatura de Psicología de los Recursos Humanos. 


\section{2.- Procedimiento}

Se administró un protocolo de recogida de información diseñado para este trabajo diseñado con 19 preguntas relacionadas con las funciones del área de Recursos Humanos (según Marín y Delgado, 2008) y 11 ítems relacionados con el perfil blando del Gestor en RRHH.

\section{3.- Resultados}

A continuación exponemos los resultados obtenidos en esta investigación. En primer lugar, se especifican los datos obtenidos en la concepción del modelo que percibe el alumnado en función de la titulación de origen; en segundo lugar, se describen los resultados hallados en relación a la percepción del alumnado sobre las 9 funciones de los RRHH según la clasificación de Martín y Delgado (2008); y, tercer lugar, se exponen los datos obtenidos en relación a la opinión que tiene el alumnado en cuanto a las competencias necesarias (perfil blando) para desempeñar adecuadamente la gestión de RRHH.

a) Concepción del Departamento en Linea o Staff

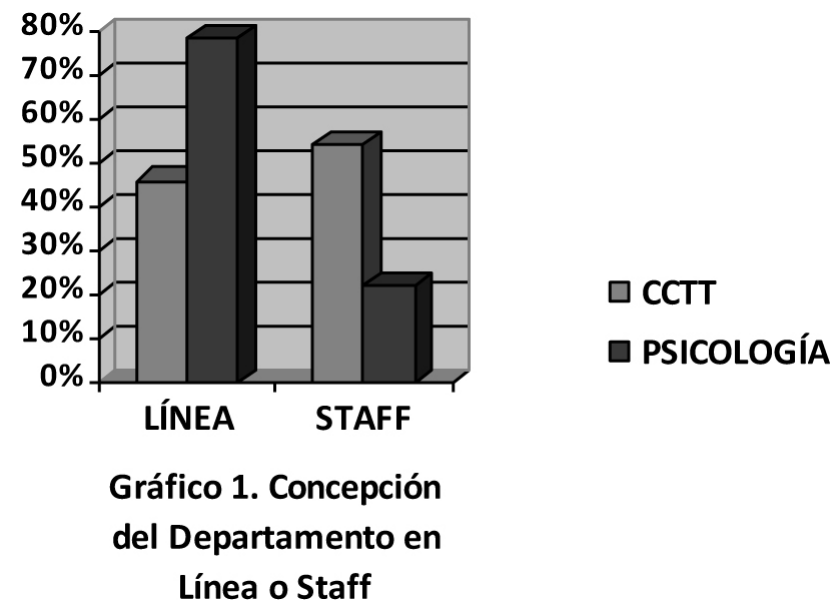

Los resultados indican que el 46\% del alumnado de CCTT entienden el Departamento de RRH como un Departamento en Línea frente al 78\% del alumnado de Psicología; así mismo, el 54\% del alumnado de CCTT concibe el Departamento de RRHH como un Departameto Staff frente al 22\% del alumnado de Psicología. 
b) Funciones del Área de los RRHH

\section{Función Estratégica}

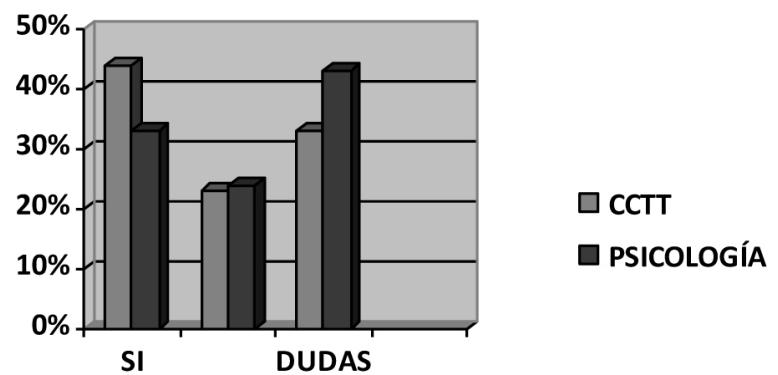

\section{Gráfico 2. Función Estratégica}

Los resultados informan que en relación al alumnado de CCTT, el 44\% considera que esta función es propia del gestor de los RRHH, el 23\% opina que no y el 33\% no lo tienen claro. En relación al alumnado de la Titulación de Psicología, el 33\% considera que es una función del Gestor de los RRHH, un 24\% considera que no y el $43 \%$ lo duda.

\section{Función Analítica}

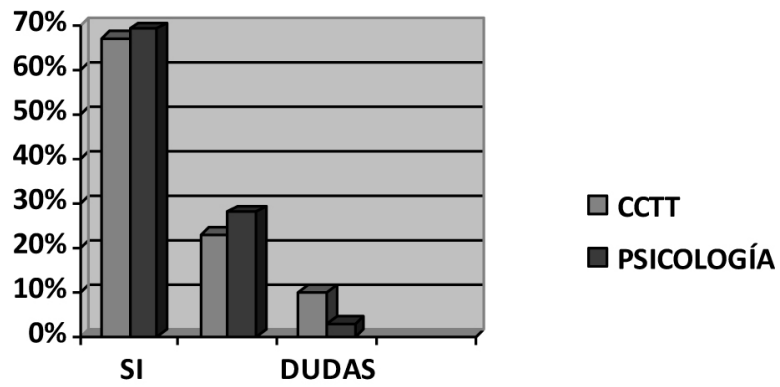

Gráfico 3. Función Analítica

Con respecto a esta función, el $67 \%$ del alumnado de CCTT, considera que es propia del gestor de los RRHH, el 23\% opina que no y el 10\% no lo tienen claro. En cuanto al alumnado de la Titulación de Psicología, el 69\% opina que es una función del Gestor de los RRHH, un 28\% piensa lo contrario y el 3\% lo duda. 
Función de Vinculación y Desvinculación

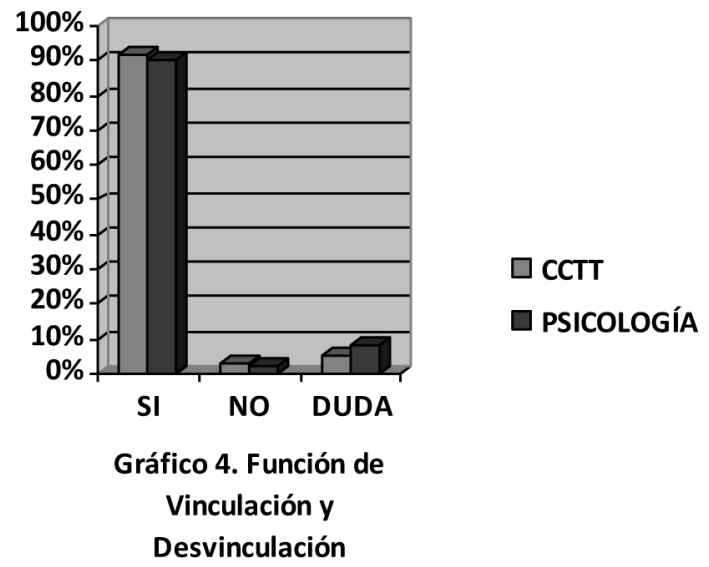

Los datos obtenidos resultados informan que en relación al alumnado de CCTT, el 92\% considera que esta función es propia del gestor de los RRHH, el 3\% opina que no y el 5\% no lo tienen claro. En relación al alumnado de la Titulación de Psicología, el 90\% considera que es una función del Gestor de los RRHH, un 2\% considera que no y el 8\% lo duda.

Función de Desarrollo

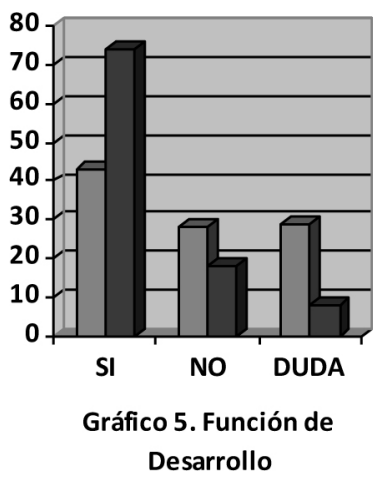

\section{$\square$ CCTT \\ $\square$ PSICOLOGÍA}

Los resultados informan que en relación al alumnado de CCTT, el 43\% considera que esta función es propia del Gestor de los RRHH, el 28\% opina que no y el 29\% no lo tienen claro. Con respecto al alumnado de la Titulación de Psicología, el 74\% piensa que es una función del Gestor de los RRHH, un 18\% estima que no y el 8\% lo duda. 
Función de Gestión del Presupuesto y de Compensación

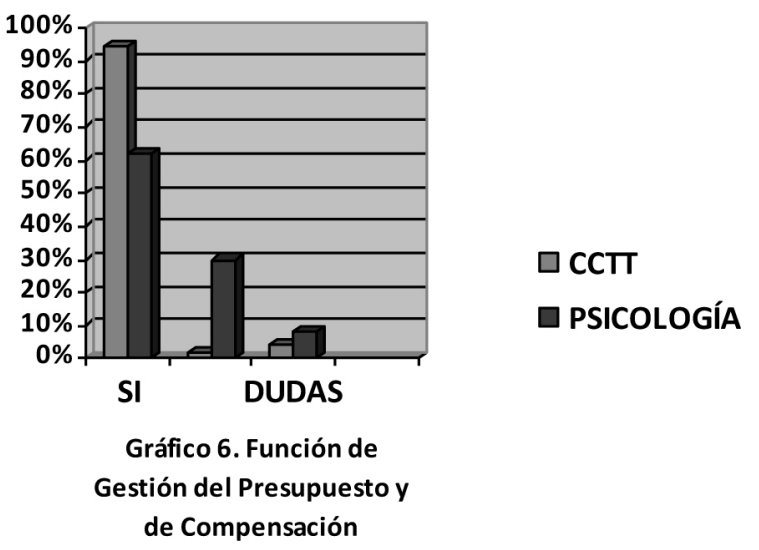

El análisis de los datos acerca de esta función manifiestan que el 94\% del alumnado de Ciencias del Trabajo estima que la Función de Gestión del Presupuesto y de Compensación es propia del Gestor de Recursos Humanos, un 2\% considera que no y un $4 \%$ tiene dudas. En cuanto al alumnado de Psicología, el 62\% informa que es una función de los RRHH, mientras que un 30\% considera que no y un $8 \%$ presenta dudas.

Función de Relacionales Laborales

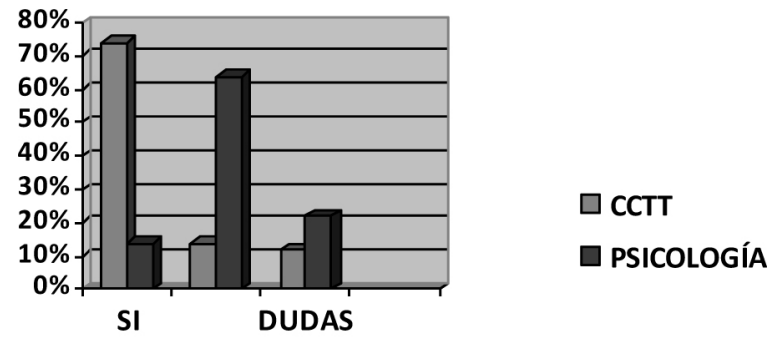

Gráfico 7. Función de

Relaciones Laborales

Los resultados indican que en relación al alumnado de CCTT, el 74\% considera que esta función es propia del Gestor de los RRHH, el 14\% opina que no y el 12\% no lo tiene claro. Con respecto al alumnado de la Titulaciín de Psicología, el 14\% considera que es una función del Gestor de los RRHH, un 64\% consiera que no y el $22 \%$ lo duda. 
Función de Prevención de Riesgos

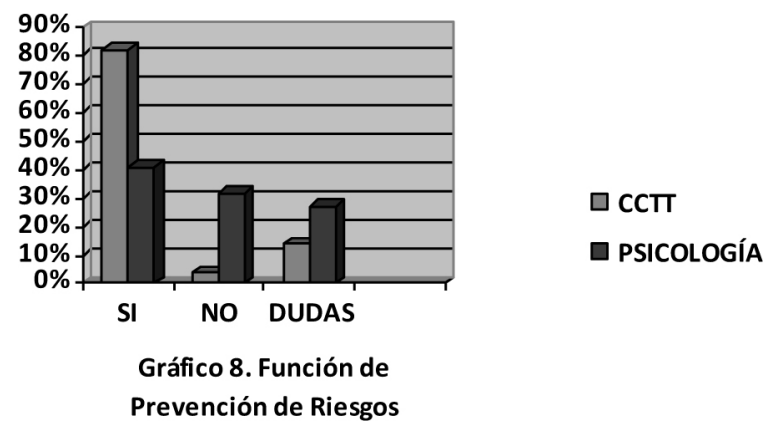

Las estimaciones encontradas en esta función indican que, el 82\% del alumnado de CCTT informa que es propia del Gestor de RRHH, frente a un 4\% que opina lo contario y el 14\% que presenta dudas. Así mismo, el 41\% del alumnado de Psicología manifiesta que es una función propia del Gestor de RRHH, un 32\% no lo considera y un $27 \%$ no lo tiene claro.

Función de Gestión del Proceso y de los Resultados

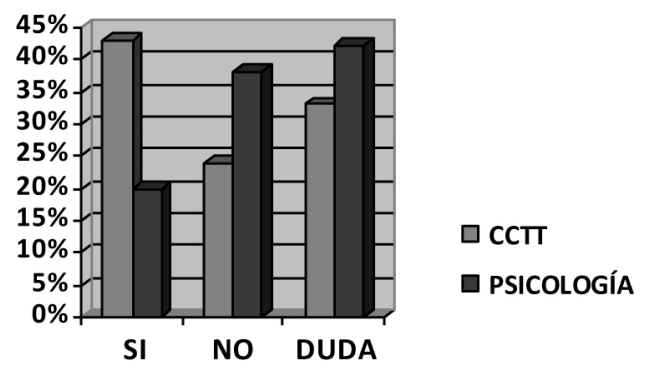

\section{Gráfico 9. Función de \\ Gestión del Proceso y \\ de los Resultados}

El análisis de los datos nos reporta que, en relación al alumnado de CCTT, el $43 \%$ estima que esta función es propia del Gestor de los RRHH, el 24\% opina que no y el 33\% no lo tiene claro. En relación al alumnado de la Titulación de Psicología, el 20\% considera que es una función del Gestor de los RRHH, un 38\% considera que no y el $42 \%$ lo duda. 


\section{Función Consultora}

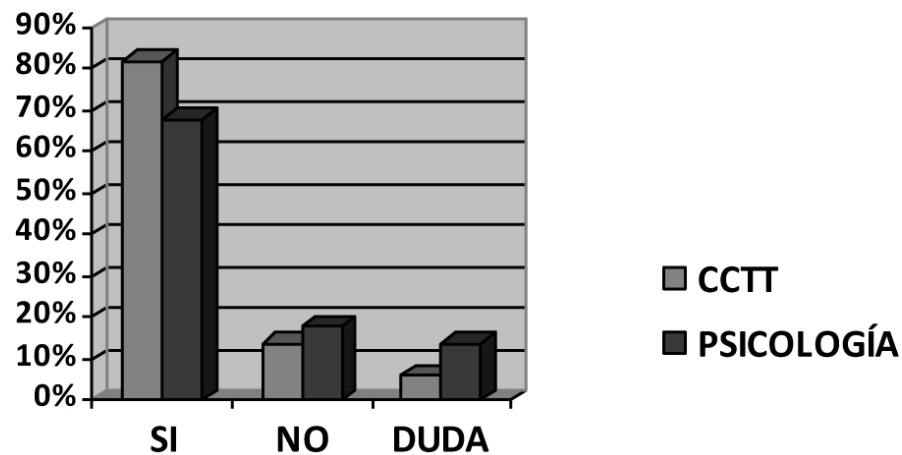

\section{Gráfico 10. Función Consultora}

Los datos hallados en esta función manifiestan que el 82\% del alumnado de CCTT considera que esta función es propia del gestor de los RRHH, el 14\% opina que no y el 6\% no lo tienen claro. En relación al alumnado de la Titulación de Psicología, el 68\% informa que es una función del Gestor de los RRHH, un 18\% piensa que no y el 14\% lo duda.

\section{c) Perfil necesario para ser un buen Gestor de los RRHH}

En relación al perfil de competencias que es considerada más adecuada para el profesional de los RRHH, los resultados informan que existe una mayor tendencia del alumnado de Psicología a enfatizar las competencias relacionadas con la empatía (44\% frente a 88\%), orientación hacia el cliente (54\% frente a 77\%), coaching (68\% a 92\%), orientación (75\% a 92\%) y gestión de personas (78\% a 96\%). De la misma forma, se observa una mayor tendencia del alumnado de CC del Trabajo a enfatizar la orientación hacia los resultados ( $82 \%$ frente a $58 \%$ ), perspectiva organizacional (84\% a 65\%), visión de negocio (87\% frente a 59\%), análisis financiero y planificación (79\% frente a 34\%).

De la misma forma, se observan resultados muy parecidos en competencias como Habilidades de Comunicación (98\% a 99\%) y Trabajo en Equipo (98\% a 99\%). 


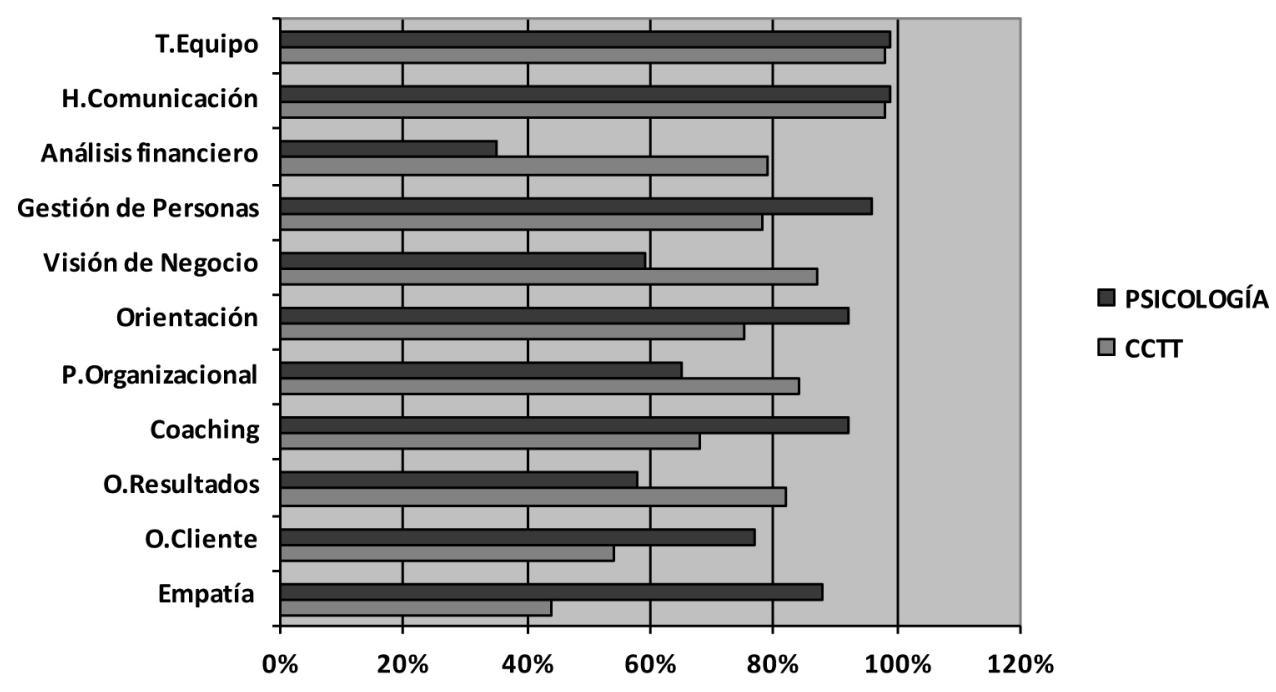

Gráfico 11. Perfil Gestor de RRHH

\section{4.- CONCLUSIONES Y DISCUSIÓN}

En relación a las diferencias encontradas (46\% frente a 78\%) relacionadas con el modelo de RRHH que concibe el alumnado (en Linea o Staff), es relevante observar cómo son los alumnos de Psicología los que manifiestan una concepción más integrada de la Gestión de los RRHH dentro de la organización. Desde el análisis de estos resultados, que para nada deben ser motivos de generalización, los alumnos de Psicología entienden el Departamento como una parte de la institución que es transversal a todas y en donde se involucran a todos los departamentos y personas que tienen responsabilidades sobre un equipo humano. De esta forma, y en la línea de algunos autores como Cascio (1998), entienden la dirección de los RRHH como una responsabilidad que debe ser asumida por todos los directivos que gestionan una organización más que por un departamento específico para este objetivo. De otra parte, un gran número de alumnos de CCTT, lo conciben como un Departamento más estanco que tiene como finalidad principal asesorar y servir de consultor interno de las otras áreas de la organización que realizan las actividades básicas de la misma. Es decir, lo entienden como un área especializada en esta responsabilidad y que, por tanto, es la encargada de respuesta a las demandas ocasionadas de la gestión de las personas que componen la organización. 
Con respecto a las diferencias encontradas en las diversas funciones, se comentarán a continuación, dado las diferencias encontradas, las relacionadas con la Gestión del Presupuesto y de Compensación, del Desarrollo, de Relaciones Laborales, de Prevención de Riesgos, de Gestión del Proceso y de los Resultados:

En relación a la función de Gestión del Presupuesto y de Compensación, se han encontrado una gran diferencia en los datos analizados, siendo el $94 \%$ del alumnado de CCTT frente al 62\% del alumnado de Psicología. Según estos hallazgos, estos últimos entienden que aquellas actividades relacionadas con el sistema salarial, administración de salarios e incentivos y beneficios económicos o sociales no son competencia del gestor de los RRHH. Estos resultados pueden ser indicadores de que el concepto de RRHH que prevalece en esta Titulación, como se puede observar en los datos obtenidos en la Función de Desarrollo, se entiende más como un concepto de orientador o coaching. De esta forma, las funciones que tienen que ver con las retribuciones son entendidas como responsabilidad "del jefe", considerándolo como una persona que prevalece en un orden superior, es decir, con mayor jerarquía, y que es el que determinará dicho aspecto de su puesto de trabajo. Podemos observar, por tanto, y de una forma paradójica, que a pesar de haber obtenido datos en los que el alumnado de Psicología entendía este Departamento como un modelo en línea, sin embargo, conserva aún la reminiscencia de las políticas más verticales existente a lo largo de la historia de las organizaciones en donde lo referente a las retribuciones era motivo de conflictos, poco negociable y quedaba en manos de los dueños de las empresas.

En cuanto a la Función del Desarrollo relacionado con las tareas de orientación, formación, desarrollo, evaluación del desempeño y planes de carrera, se observa como existe una gran diferencia entre los alumnos de ambas titulaciones, $43 \%$ frente a $73 \%$. La explicación a estos resultados podemos encontrarlo en que se trata de una función muy enfocada en la atención a la persona. Como se ha comentado anteriormente, el alumnado de Psicología entiende al Gestor de RRHH como un profesional que debe centrar su tarea más en el concepto de coach, orientador o de desarrollo de habilidades, entre otros aspectos. Más específicamente, entiende que el Gestor de RRHH debe enfocar sus actuaciones en los aspectos psicosociales de la persona y, no tanto, a otras variables de la organización. Así, nuevamente podemos observar que, existe cierta tendencia a inclinar las acciones del profesional de los RRHH en función a la formación de base. La Titulación de Psicología, centra sus objetivos en el desarrollo de la persona y en la mejora de su calidad de vida. Este hecho carece de importancia siempre que el alumnado entienda que la optimización de los resultados de una organización, no sólo dependen de esta variable, aunque sea de gran relevancia, sino que forma parte de un conjunto.

En relación a las funciones de Relaciones Laborales, Prevención de Riesgos y Gestión del Proceso y de los Resultados, se observa nuevamente como existe diferencias 
relevantes entre el alumnado de amas titulaciones. Así, estas tres funciones son más contempladas por el alumnado de CCTT como parte del conjunto de actividades del gestor de RRHH. Se hace evidente cómo los modelos aprendidos por los alumnos tienen matices diferentes. La Titulación de RRHH y RRLL tiene como característica estar conformada por un conjunto de profesionales de diferentes disciplinas, este aspecto tiene como ventaja una percepción de la especialización de los RRH mucho más amplia. En contraposición, el alumnado de Psicología recibe una formación más centrada en la persona, y no en los resultados que, como apuntan las últimas investigaciones, son un recurso necesario para la optimización de las organizaciones.

Finalmente, en lo referente al perfil del profesional de los RRHH, resultan de interés los datos obtenidos en las competencias de Habilidades de Comunicación (98\% en CCTT a 99\% en Psicología) y el Trabajo en Equipo (98\% en CCTT a 99\% en Psicología) en ambas titulaciones. No es difícil encontrar en ofertas de empleo o currículums vitaes competencias transversales tales como Habilidades de Comunicación y de Trabajo en Equipo. En los últimos años, con la configuración de los nuevos planes de estudio según el Plan Bolonia dentro del Marco Europeo de Educación Superior, se ha hecho visible la importancia de la formación en competencias. Esta circunstancia, a la que se le añade la incorporación que los docentes han hecho de las mismas en sus guías docentes ha conllevado como consecuencia, generar la preocupación del alumnado entendiendo que son herramientas necesarias e imprescindibles para poder abordar su futura empleabilidad teniendo en cuenta las exigencias del mercado laboral. Evidencia de este hecho se puede observar en los resultados obtenidos relacionados con el perfil del Gestor de RRHH, en donde los porcentajes relacionados con estas dos competencias transversales, son prácticamente equitativos. De la misma forma, se observa como la competencias más relacionadas con la persona como coach, empatía, orientación al cliente son más valoradas en el alumnado de la Titulación de Psicología mientras que las más orientadas a la visión empresarial como visión de negocio, orientación de resultados o análisis financiero son más valoradas por el alumnado de CCTT.

Como reflexión final sería interesante llevar a cabo una crítica relacionada con el procedimiento de los planes de estudio de los nuevos grados. Es importante destacar que, sin dar lugar a generalizaciones, muchos de ellos se han generado al amparo de luchas internas departamentales que han traído como consecuencia que "gane el más fuerte" obviando la verdadera calidad docente y necesidad de los futuros profesionales. En este "campo de batalla" es evidente que, no sólo ha perdido el alumnado, que debe recibir la formación que los gestores de su conocimientos han creído conveniente, sino que se han denostado campos de especialización como los Recursos Humanos, que poseen la riqueza de ser "tierra de nadie" o "tierra de todos" y, por tanto, debe estar formada por aportaciones de múltiples áreas de conocimiento. Paradójicamente, a pesar de los mensajes enviados en los últimos años de la necesidad de generar espacios de interdisciplinariedad, multidisciplinariedad, etc. algunos docentes 
universitarios se mantiene reticentes y siguen permaneciendo en departamentos o áreas estancos y, en muchos ocasiones, son intrusos de otras áreas de conocimientos que no les competen, por el simple hecho de no entender que "todos los saberes no son de ellos". Probablemente la reflexión deba pasar o partir por analizar este tipo de docente y quizás deban ser los gestores de las instituciones las que generen una cultura organizacional que permita la prevención de estos sucesos y mayores espacios de diálogos entre todos los componentes que lo conforman fomentando un mejor clima laboral y, en consecuencia, una docencia de mayor calidad.

\section{5.- REFERENCIAS BIBLIOGRÁFICAS}

Albizu, E y Landaeta, J. (2001): Dirección Estratégica de los Recursos Humanos, Madrid, Editorial Pirámide.

Alles, M. (2008): Desarrollo del Talento Humano, Argentina, Ediciones Granica.

Bohlander G., Sherman A. Snell S. (2003): Administración de recursos humanos, España, Internacional Thompson Ediciones Spain.

Cascio, W.F. (1995): "Whither Industrial and Organizacional Psychology in a changing world of work?", American Psychologist, 50, pp. 928-939.

Cascio, W.F. (1998): Managing human resources: Productivity, quality of work life, profits, Nueva York, McGraw-Hill.

Besseyre Des Horst, C.H. (1988): Vers une gestion estratégique des ressources humaines, París, Les Editions d'Organizations.

Dollan, S., Shuler, R. y Valle, R. (1999): La gestión de los recursos humanos, Madrid, MacGraw-Hill Interamericana.

Gómez-Mejía, L. R; Balkin, D. B.; Cardy, R. L. (1999): Gestión de los Recursos Humanos, Madrid, Prentice-Hall.

Gómez-Mejía, L. R; Balkin, D. B.; Cardy, R. L. (2001): Dirección y gestión de recursos bumanos, Madrid, Prentice-Hall.

Gónzalez Fernández, L. y de De Elena, J. (1998): "Propuesta de un modelo de Dirección y Gestión de Recursos Humanos”, Estudios Financieros: Revista de Trabaio y Seguridad social, 189, pp. 95-150.

Ivancevich, J.M. (1998): Human Resource management, New York, McGraw-Hill.

Martín-Quiros y Delgado (2008): "La dirección y gestión de los recursos humanos en las organizaciones", Rodríguez, A. y Zarco. V. (dirs), Psicología de los Recursos Humanos, Madrid, Ed. Pirámide, pp.17-39. 
Milckovich, G. T. y Boudreau, J., W. (1994): Dirección y administración de recursos bumanos, EEUU, Addison-Wesley Iberoamericana.

Peiró, J.M. (1999): "El modelo "AMIGO", Marco Contextualizador del Desarrollo y la Gestión de Recursos Humanos”, Papeles del Psicólogo, 72, pp. 3-15.

Werther, W. y Davis K. (1991): Administración de Personal y Recursos Humanos. México, Editorial MC. Graw-Hill. 